\title{
INFLUÊNCIA DA TEMPERATURA NO RENDIMENTO DOS PRODUTOS DA CARBONIZAÇÃO DE Eucalyptus microcorys
}

\author{
Renato da Silva Vieira ${ }^{1}$, José Tarcísio Lima², Thiago Campos Monteiro \\ Thaisa de Sousa Selvatti ${ }^{4}$, Edy Eime Pereira Baraúna ${ }^{1}$, Alfredo Napoli ${ }^{5}$,
}

(recebido: 10 de novembro de 2009; aceito: 28 de setembro de 2012)

\begin{abstract}
RESUMO: Durante a produção de carvão vegetal, diferentes produtos são formados. Esses produtos são resultantes, principalmente, da temperatura final de carbonização. Sendo o carvão vegetal um dos principais insumos na produção de ferro gusa no Brasil, no presente trabalho, objetivou-se avaliar a influência da temperatura final de carbonização no rendimento dos produtos gerados, e também verificar a influência da posição radial e longitudinal de amostragem nos rendimentos de cada produto. Para isso, foram retiradas amostras da posição interna e externa ao longo do raio e também de três diferentes alturas a partir de quatro árvores de Eucalyptus microcorys. As amostras foram carbonizadas em forno elétrico experimental adaptado, com condensador resfriado à água e frasco coletor de materiais voláteis condensáveis. As temperaturas finais de carbonização foram de $500,600,700,800$ e $900^{\circ} \mathrm{C}$. Em seguida, foram calculados os rendimentos gravimétricos em carvão, líquido pirolenhoso e gases não condensáveis. Os resultados mostram que não houve diferença de rendimento gravimétrico em carvão nas posições longitudinais e radiais estudadas; o rendimento em líquido pirolenhoso e gases não condensáveis apresentaram variações nas temperaturas de $700^{\circ} \mathrm{C}$ e $800^{\circ} \mathrm{C}$; a variação do rendimento gravimétrico em carvão, entre as temperaturas de $500^{\circ} \mathrm{C}$ a $900^{\circ} \mathrm{C}$ foi de $15 \%$; a variação do rendimento em líquido pirolenhoso no sentido radial de amostragem foi, em média, de 8\%; a variação do rendimento em gases não condensáveis no sentido radial de amostragem foi, em média, de $16 \%$.
\end{abstract}

Palavras-chave: Rendimento gravimétrico, líquido pirolenhoso, gases não condensáveis, variação radial, carvão vegetal.

\section{INFLUENCE OF TEMPERATURE ON PRODUCTS YIELD OF Eucalyptus microcorys CARBONIZATION}

\begin{abstract}
During charcoal production different products are formed. These products are influenced primarily by the temperature of carbonization. Given that charcoal is the main input in the production of pig iron in Brazil, this study evaluated the influence of final temperature of carbonization of the products generated and also the influence of the radial and longitudinal sampling on the yield of each product. Samples were taken from internal and external position along the radius and also from three different heights from four Eucalyptus microcorys trees. The samples were carbonized in an electric furnace with an experimental water-cooled condenser and a collecting bottle of condensable volatile materials. The final temperatures of carbonization were $500,600,700,800$ and $900^{\circ} \mathrm{C}$. The gravimetric yield, tar and non-condensable gases were calculated. The results showed no difference in the gravimetric yield in the longitudinal and radial positions studied, while the tar yield and non-condensable gases showed temperature variations of $700^{\circ} \mathrm{C}$ and $800^{\circ} \mathrm{C}$ and the variation of the gravimetric yield temperatures between $500^{\circ} \mathrm{C}$ to $900^{\circ} \mathrm{C}$ was $15 \%$, the change of yield of tar from the radial direction of sampling was on average $8 \%$, the variation of the yield of non-condensable gases in a radial sampling was on average $16 \%$.
\end{abstract}

Key words: Gravimetric yield, tar, non condensable gases, radius variation, charcoal.

\section{INTRODUÇÃO}

O Brasil é um dos maiores produtores de ferro gusa a carvão vegetal do mundo. Segundo Milanez (2009), o país ocupa a $11^{\mathrm{a}}$ posição no mercado mundial na exportação de aço bruto que usa, geralmente, o coque nos altos-fornos. Além disso, existe uma política governamental no sentido de aumentar a produção de aço no país, tanto para atender

\footnotetext{
${ }^{1}$ Engenheiro Florestal, Professor Doutor em Ciência e Tecnologia da Madeira - Universidade Federal do Tocantins/UFT - Departamento de Engenharia Florestal - 77402-970 - Gurupi, TO, Brasil - rsvieira@uft.edu.br, ebarauna@uft.edu.br

${ }^{2}$ Engenheiro Florestal, Professor Doutor em Ciências Florestais - Universidade Federal de Lavras/UFLA - Departamento de Ciências Florestais - Cx. P. 3037 - 37200-00 - Lavras, MG, Brasil - jtlima@ufla.br

${ }^{3}$ Engenheiro Florestal, Professor Doutorando em Ciência e Tecnologia da Madeira - Universidade Federal do Mato Grosso do Sul/UFMS Cx. P. 112 - 79560-000 - Chapadão do Sul, MS, Brasil - tcmforest@yahoo.com

${ }^{4}$ Engenheira Florestal - Universidade Federal de Lavras/UFLA - Departamento de Ciências Florestais - Cx. P. 3037 - $37200-00$ Lavras, MG, Brasil - thausaselvatti@gmail.com

"Graduado em Ciências dos Materiais, Pesquisador Doutor em Sistemas Energéticos - CIRAD - Département "PERSYST" Performance des Systèmes de production et de transformation tropicaux - TA B-40/16 73 - Rue Jean-François Breton 34398 - Montpellier, France alfredo.napoli@cirad.fr
}

Cerne, Lavras, v. 19, n. 1, p. 59-64, jan./mar. 2013 
demandas domésticas como o mercado externo (ALENCAR; SOFIA, 2009).

Para a produção adequada de ferro gusa é necessário, além de outros fatores, minério de ferro e carvão de qualidade. O carvão vegetal representa cerca de $40 \%$ dos custos de produção na indústria siderúrgica (SANTOS, 2008). Um dos problemas enfrentados pela indústria siderúrgica brasileira é a heterogeneidade do carvão vegetal utilizado na fabricação do aço em termos de suas características físicas, químicas e mecânicas e o baixo rendimento nos processos de carbonização utilizados atualmente. Os rendimentos e a heterogeneidade do carvão vegetal são decorrente das variações do lenho e do processo de carbonização.

Quanto à matéria-prima utilizada para a produção de carvão vegetal no Brasil, a madeira de Eucalyptus apresenta destaque. $\mathrm{O}$ setor florestal brasileiro conta com, aproximadamente, 4,8 milhões de hectares com plantios desse gênero (ASSOCIAÇÃO BRASILEIRA DE PRODUTORES DE FLORESTAS PLANTADAS - ABRAF, 2012). Deste total, segundo a mesma fonte, o segmento de siderurgia a carvão vegetal, em 2011, consumiu quase 17 milhões de $\mathrm{m}^{3}$ de toras de Eucalyptus.

A maior parte da madeira utilizada para a carbonização é cortada em plantios com seis ou sete anos, idade determinada de acordo com aspectos econômicos. Apesar das propriedades da madeira exercerem influência sobre a qualidade do carvão vegetal em aspectos como porosidade, composição química, densidade, poder calorífico, entre outros (OLIVEIRA et al., 1982; SANTOS, 2008; VITAL et al., 1989), muitas vezes elas não são consideradas na determinação da idade de corte.

Outro fator que contribui no rendimento da carbonização e na heterogeneidade química do carvão vegetal são as características do processo de carbonização como a temperatura de aquecimento e a temperatura final da carbonização. A variação da temperatura dentro dos fornos produz uma matéria-prima com teor de carbono fixo, próximo de $98 \%$ e uma madeira torrificada, em que o processo de carbonização não se completou (PINHEIRO et al., 2005). A temperatura final de carbonização afeta as propriedades do carvão e os rendimentos, tendo alguns estudos avaliado esse parâmetro. Trugilho e Silva (2001) avaliaram as influências de temperaturas entre $300-900^{\circ} \mathrm{C}$ no carvão vegetal de Jatobá (Himenea courbaril) e obtiveram diferenças no rendimento em carbono fixo, material volátil, teor de cinzas e outros em função da temperatura final de carbonização; Pinheiro et al. (2005) estudaram as influências de temperaturas entre $200-650^{\circ} \mathrm{C}$ e taxa de aquecimento nas propriedades do carvão vegetal de Eucalyptus microcorys e obtiveram a temperatura ótima do processo entre $300-450^{\circ} \mathrm{C}$ para os rendimentos gravimétricos em carvão e teor de carbono fixo.

A variação da temperatura final de carbonização pode ser decorrente das características geométricas dos fornos e do leito da madeira (FÁVERO et al., 2007), assim como à velocidade de carbonização, campo de escoamento dos gases, além das características da biomassa como umidade, diâmetro, comprimento e posição (PINHEIRO et al., 2005).

O presente trabalho pertence a um estudo sobre a influência da madeira obtida com árvores de idade acima da utilizada no setor florestal brasileiro para os plantios de Eucalyptus e com parâmetros de carbonização similares à produção de coque com carvão fóssil, ou seja, até altas temperaturas $\left(>900^{\circ} \mathrm{C}\right)$. Dessa forma, no presente trabalho, objetivou-se avaliar a influência da temperatura final de carbonização no balanço de massa da carbonização, ou seja, no rendimento dos produtos gerados nesse processo, e também verificar a influência da posição radial e longitudinal de amostragem nos rendimentos dos produtos da carbonização.

\section{MATERIAL E MÉTODOS}

Foram utilizadas quatro árvores de Eucalyptus microcorys oriundas de um plantio de procedência implantado na Universidade Federal de Lavras (UFLA). As árvores abatidas apresentavam idade média de 35 anos. A altura média das árvores foi de 30 metros e o diâmetro médio de $450 \mathrm{~mm}$, obtidos na altura de 1,3 metros de solo. A densidade básica média do lenho foi de $0,880 \mathrm{~g} / \mathrm{cm}^{3}$.

Depois de abatidas, as árvores foram seccionadas no campo em três toras de 5 metros de comprimento. Estas foram transportadas para a Unidade Experimento de Desdobro e Secagem da Madeira da UFLA, onde foram desdobradas em pranchas centrais de $10 \mathrm{~cm}$ de espessura.

A partir das pranchas centrais foram confeccionados corpos-de-prova de dimensões de 5 x 5 x $21 \mathrm{~cm}$, considerando as posições radiais como externa e interna.

As carbonizações foram realizadas no Laboratório de Energia da Biomassa da UFLA, utilizando um forno elétrico (mufla) adaptado com condensador resfriado à água e frasco coletor (kitasato) de materiais voláteis condensáveis, apresentado na Figura 1.

Cerne, Lavras, v. 19, n. 1, p. 59-64, jan./mar. 2013 


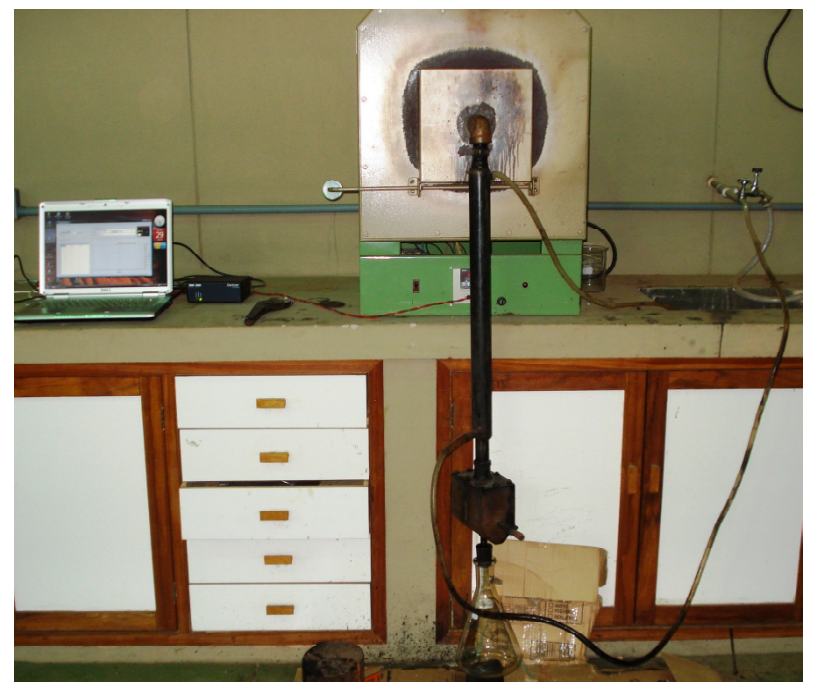

Figura 1 - Forno elétrico adaptado com condensador resfriado à água e frasco coletor de materiais voláteis condensáveis, juntamente com o regulador de temperatura informatizado.

Figure 1 - Electric furnace fitted with water cooled condenser and bottle collector of volatile condensable materials along with computerized temperature control.

Os parâmetros de carbonização utilizados foram taxa de aquecimento de $0,5^{\circ} \mathrm{C} / \mathrm{min}$ e as temperaturas finais de carbonização de $500{ }^{\circ} \mathrm{C}, 600{ }^{\circ} \mathrm{C}, 700{ }^{\circ} \mathrm{C}, 800{ }^{\circ} \mathrm{C}$ e $900{ }^{\circ} \mathrm{C}$. Esses parâmetros eram enviados ao forno elétricos por um regulador de temperatura informatizado (PID) de modo que, ao atingir a temperatura desejada, o fornecimento de energia era imediatamente desligado. Essas temperaturas consideram a faixa real de temperaturas em fornos de alvenaria, que apresentam o gradiente térmico gerado pelo uso de sistema de combustão parcial, e para iniciar os estudos sobre a coqueficação do carvão vegetal, processo que ocorre em temperatura elevada $\left(900^{\circ} \mathrm{C}\right)$.
Para os cálculos de rendimento, a madeira foi seca, pesada e colocada no interior do reator para proceder a carbonização e, em seguida, o carvão produzido e o líquido pirolenhoso resultante da condensação dos gases da carbonização eram pesados. A quantificação dos gases que não se condensavam foi feita por diferença. Os cálculos dos rendimentos foram feitos por meio das seguintes equações:

$R C=\frac{\text { Ps carvão }}{\text { Ps madeira }} \times 100$

em que:

RC- Rendimento gravimétrico do carvão (\%);

Ps carvão- peso seco do carvão (g);

Ps madeira- peso seco da madeira $(\mathrm{g})$.

$R L=\frac{P \text { líquido }}{\text { Ps madeira }} \times 100$

em que:

RL- Rendimento em líquido pirolenhoso (\%);

P líquido- peso líquido condensado (g);

Ps madeira- peso seco da madeira (g).

$R G=100-(R C+R L)$

em que:

RG- Rendimento em gás não condensável (\%);

RC- Rendimento gravimétrico do carvão (\%);

RL-Rendimento em líquido pirolenhoso (\%).

\section{RESULTADOS E DISCUSSÃO}

Na Tabela 1, estão apresentadas as médias e os coeficientes de variação dos rendimentos gravimétrico em carvão, líquido pirolenhoso e gases não condensáveis, para cada uma das temperaturas finais de carbonização.

Tabela 1 - Rendimentos dos produtos da carbonização obtidos nas diferentes temperaturas.

Table 1 - Yields of products obtained at different carbonization temperatures.

\begin{tabular}{|c|c|c|c|c|c|c|}
\hline \multirow{3}{*}{ Temperatura ${ }^{\circ} \mathrm{C}$} & \multicolumn{6}{|c|}{ Rendimento Gravimétrico } \\
\hline & \multicolumn{2}{|c|}{ Carvão vegetal } & \multicolumn{2}{|c|}{ Líquido pirolenhoso } & \multicolumn{2}{|c|}{ Gases não condensáveis } \\
\hline & Média & $\mathrm{CV}$ & Média & $\mathrm{CV}$ & Média & $\mathrm{CV}$ \\
\hline 500 & 34 & 6 & 43 & 13 & 23 & 26 \\
\hline 600 & 31 & 10 & 40 & 22 & 29 & 31 \\
\hline 700 & 28 & 8 & 49 & 4 & 22 & 9 \\
\hline 800 & 27 & 7 & 48 & 7 & 24 & 17 \\
\hline 900 & 29 & 6 & 42 & 25 & 29 & 36 \\
\hline
\end{tabular}

M \% - média dos rendimentos em porcentagem; CV \%-Coeficiente de variação em porcentagem. 
Observa-se, na Tabela 1 , o rendimento da carbonização entre $500^{\circ} \mathrm{C} \mathrm{e} 900^{\circ} \mathrm{C}$. Os rendimentos em carvão estão na faixa de 29 e $34 \%$. Os resultados indicam que a idade das árvores possui pouca influência no rendimento médio em carvão. Por outro lado, observa-se que a queda do rendimento gravimétrico em carvão da temperatura final de carbonização de $500^{\circ} \mathrm{C}$ para $900^{\circ} \mathrm{C}$ foi de $5 \%$ e que de $700^{\circ} \mathrm{C}$ até $900^{\circ} \mathrm{C}$ ocorreu uma tendência de estabilização do rendimento gravimétrico. Esse resultado indica que a transformação da madeira em carvão vegetal ocorre durante a primeira etapa da carbonização, até $500^{\circ} \mathrm{C}$. E que entre $500^{\circ} \mathrm{C}$ e $900^{\circ} \mathrm{C}$ a transformação do carvão vegetal ocorre, principalmente, com o rearranjo da estrutura do carvão vegetal. O mesmo fenômeno acontece com o coque.

Resultados semelhantes foram os obtidos por Oliveira et al. (1982), que avaliaram os rendimentos gravimétricos em diferentes temperaturas de carbonização, utilizando Eucalyptus grandis com 5,5 anos e Trugilho e Silva (2001), que também avaliaram os rendimentos gravimétricos em diferentes temperaturas de carbonização, utilizando Jatobá (Himenea courbaril L.) na mesma faixa de $500^{\circ} \mathrm{C}$. Ambos os autores observaram a diminuição do rendimento gravimétrico em carvão com o aumento da temperatura final de carbonização, sendo que Trugilho e Silva (2001) ainda observaram estabilização desses rendimentos nas temperaturas mais elevadas.

É importante ressaltar que o estudo com temperaturas que não são aplicadas no mercado vem ao encontro de novas tecnologias que estão sendo estudadas para a produção de carvão com, as quais, os fornos mantêm as temperaturas acima de $500^{\circ} \mathrm{C}$. Esse parâmetro, produz carvão com rendimento satisfatório e com resistência mecânica desejável para a aplicação siderúrgica.

O rendimento em líquido pirolenhoso e gases não condensáveis inicialmente tenderam a aumentar, porém, em seguida, não seguiram uma tendência, diferindo de Valente et al. (1985), que avaliaram os rendimentos desses produtos na carbonização de Eucalyptus desses produtos entre $300^{\circ} \mathrm{C} \mathrm{e} 600^{\circ} \mathrm{C}$ e observaram uma estabilização após $450^{\circ} \mathrm{C}$. Entretanto, Oliveira et al. (1982), de uma maneira geral, encontraram maiores rendimentos de liquido pirolenhoso e gases não condensáveis, à medida que a temperatura aumentava.

Cerne, Lavras, v. 19, n. 1, p. 59-64, jan./mar. 2013
Na Figura 2, verifica-se que os rendimentos entre as posições, interna e externa tendem a não se diferenciarem para o rendimento gravimétrico em carvão, exceto nas temperaturas de $700^{\circ} \mathrm{C}$ e $800^{\circ} \mathrm{C}$, resultado diferente do encontrado por Trugilho e Silva (2001) que obtiveram comportamento distinto entre cerne e alburno, de acordo com a temperatura final. Observando o rendimento em líquido pirolenhoso e gases não condensáveis há uma tendência de diferenciação a partir de $600^{\circ} \mathrm{C}$, igualando novamente em $900^{\circ} \mathrm{C}$. A diferença entre a posição interna e externa para o rendimento em líquido pirolenhoso à $700^{\circ} \mathrm{C}$ foi de $6 \%$ e para $800^{\circ} \mathrm{C}$ foi de $10 \%$, sendo que a posição que apresentou maior rendimento foi a externa. Houve também uma diferença no rendimento de gases não condensáveis e essa diferença foi de $16 \%$ na temperatura de $700^{\circ} \mathrm{C}$ e de $15 \%$ para a temperatura de $800^{\circ} \mathrm{C}$. A posição que apresentou maior rendimento em gases não condensáveis foi a posição interna. Pode se observar também que nas temperaturas onde houve diferenças nos rendimentos de líquido e gás, sendo que esses rendimentos são inversamente proporcionais, embora a diferença entre as posições não apresentem a mesma proporção dentre as posições. Os resultados encontrados para o liquido pirolenhoso e gases não condensáveis nas diferentes posições diferiram dos obtidos por Trugilho et al. (2005) que encontraram diferenças não significativas na posição radial.



Figura 2 - Rendimentos dos produtos da carbonização obtidos nas posições interna e externa em diferentes temperaturas.

Figure 2 - Yields of products obtained from carbonization on internal and external positions at different temperatures.

Na Figura 3, apresenta-se a variação longitudinal para o rendimento gravimétrico, rendimento em líquido pirolenhoso e rendimento em gases não condensáveis obtidos nas três toras do fuste. 

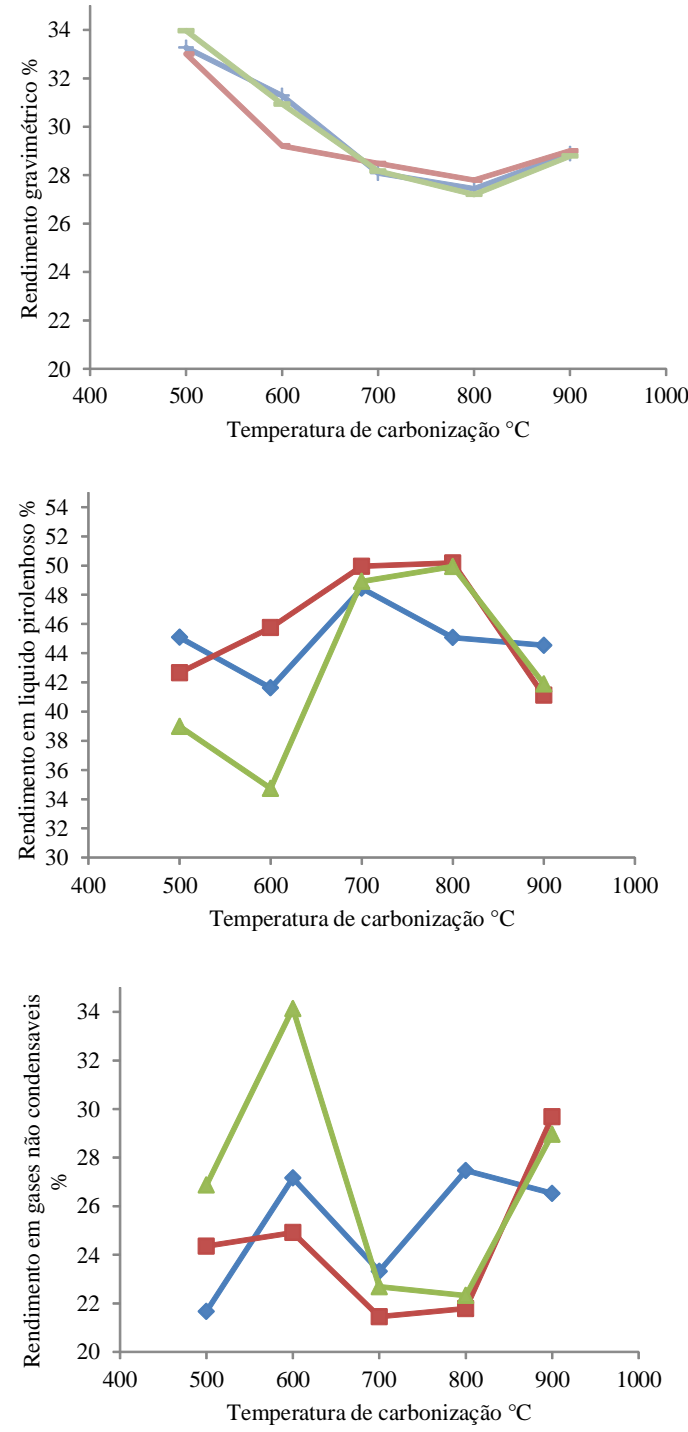

Figura 3 - A - Variação longitudinal do rendimento gravimétrico em diferentes temperaturas; B - Variação longitudinal do rendimento em líquido pirolenhoso em diferentes temperaturas; C - Variação longitudinal do rendimento em gases não condensáveis em diferentes temperaturas.

Figure 3-A - Longitudinal variation of the gravimetric yield in different temperatures; $B$ - Longitudinal variation of the pyroligneous liquid yield in different temperatures; $C$ Longitudinal variation of the non-condensable gases yield in different temperatures.

Na Figura 3A, observa-se que o rendimento gravimétrico em carvão não se altera com a diferença da posição longitudinal, no entanto, observa-se na temperatura de $600^{\circ} \mathrm{C}$ que há um decréscimo acentuado na $2^{\circ}$ tora. $\mathrm{A} 1^{\circ} \mathrm{e}$ a $3^{\circ}$ tora apresentaram tendências semelhantes.

Nas tendências do rendimento em líquido pirolenhoso (Figura 3B), em função da variação longitudinal, a $1^{\circ} \mathrm{e}$ a $3^{\circ}$ tora apresentaram menores rendimentos em líquido pirolenhoso, embora as variações apresentem magnitudes diferentes. Já, a $2^{\circ}$ tora apresentou uma tendência de aumento do rendimento até $800^{\circ} \mathrm{C}$ e, a partir de então, apresentou um decréscimo até $900^{\circ} \mathrm{C}$.

Na Figura 3C, seguindo a tendência dos outros rendimentos estudados a $1^{\circ}$ e a $3^{\circ}$ tora, apresentaram a mesma tendência para o rendimento em gases não condensáveis. Embora a $1^{\circ}$ tora, para rendimento em líquido pirolenhoso, tenha apresentado comportamento crescente, no rendimento em gases não condensáveis esse comportamento segue as $2^{\circ}$ e $3^{\circ}$ toras até $800^{\circ} \mathrm{C}$ e quando chega a $900^{\circ} \mathrm{C}$ ocorre uma inversão decrescendo o rendimento dos gases não condensáveis. A $3^{\circ}$ tora, retirada da posição mais alta do fuste, apresentou, em média, um maior rendimento em gases não condensáveis e um menor rendimento em líquido pirolenhoso. A $2^{\circ}$ tora apresentou, em média, o menor rendimento em gases não condensáveis e um maior rendimento de líquido pirolenhoso.

\section{CONCLUSÕES}

Com base nas interpretações dos resultados podese concluir que:

- não houve diferença entre os rendimentos gravimétricos em carvão nas posições longitudinais e radiais estudadas;

- os rendimentos em líquido pirolenhoso e gases não condensáveis apresentaram variações nas temperaturas de $700^{\circ} \mathrm{Ce} 800^{\circ} \mathrm{C}$;

- a variação do rendimento gravimétrico em carvão entre as temperaturas de $500^{\circ} \mathrm{Ca} 900^{\circ} \mathrm{C}$ foi de $15 \%$;

- a variação do rendimento em líquido pirolenhoso no sentido radial de amostragem foi, em média, de 8\%;

- a variação do rendimento em gases não condensáveis no sentido radial de amostragem foi, em média, de $16 \%$.

\section{REFERÊNCIAS}

ALENCAR, K.; SOFIA, J. Após pressão, Lula e Agnelli selam acordo. Disponível em: <http://www.plataformabndes.org.br/ index.php/en/noticias/38-materias/358-apos-pressao-lula-e-agnelliselam-acordo>. Acesso em: 3 out. 2009.

Cerne, Lavras, v. 19, n. 1, p. 59-64, jan./mar. 2013 
ASSOCIAÇÃO BRASILEIRA DE PRODUTORES DE FLORESTAS PLANTADAS. Anuário estatístico da ABRAF: ano base 2011. Brasília, 2012. 130 p.

FÁVERO, G. C.; VALLE, R. M.; DUARTE, T. M. Análise térmica de um sistema de combustão de alcatrão em fornos retangulares para secagem da madeira. In: CONGRESSO IBEROAMERICANO DE ENGENHARIA MECANICA, 8., 2007, Cusco. Anais... Cusco, 2007. 1 CD-ROM.

MILANEZ, B. Siderurgia, sociedade e meio ambiente. Disponível em: <http://www.riosvivos.org.br/ canal.php?c=34\&mat=12591>. Acesso em: 3 out. 2009 .

OLIVEIRA, J. B.; MENDES, M. G.; GOMES, P. A. Produção de carvão vegetal: aspectos técnicos. In: FUNDAÇÃO CENTRO TECNOLÓGICO DE MINAS GERAIS. Produção e utilização de carvão vegetal. Belo Horizonte, 1982. p. 6273.

PINHEIRO, P. C. C.; FIGUEIREDO, F. J.; SEYE, O. Influência da temperatura e da taxa de aquecimento da carbonização nas propriedades do carvão vegetal de Eucalyptus. Biomassa \& Energia, Rio de Janeiro, v. 2, n. 2, p. 159-168, 2005.
SANTOS, M. A. S. Parâmetros de qualidade do carvão vegetal para uso em alto-forno. In: FÓRUM NACIONAL SOBRE CARVÃO VEGETAL, 1., 2008, Belo Horizonte. Anais... Belo Horizonte: UFMG, 2008. 1 CD-ROM.

TRUGILHO, P. F.; SILVA, D. A. da. Influência da temperatura final de carbonização nas características físicas e químicas do carvão vegetal de Jatobá (Himenea courbaril L.). Scientia Agraria, Piracicaba, v. 2, n. 1/2, p. 45-53, 2001.

TRUGILHO, P. F.; SILVA, J. R. M.; MORI, F. A.; LIMA, J. T.; MENDES, L. M.; MENDES, L. F. B. Rendimentos e características do carvão vegetal em função da posição radial de amostragem em clones de Eucalyptus. Cerne, Lavras, v. 11, n. 2, p. 178-186, 2005.

VALENTE, O. F.; ALMEIDA, J. M.; VITAL, B. R.; DELLA LUCIA, R. M. Efeito da temperatura de carbonização nos rendimentos e propriedades do carvão vegetal produzido. Revista Árvore, Viçosa, v. 9, n. 1, p. 28-39, jan./fev. 1985.

VITAL, B. R.; ANDRADE, A. M.; VALENTE, O. F.; CAMPOS, J. C. C. Influência da casca no rendimento e na qualidade do Carvão vegetal de Eucalyptus grandis. IPEF, Piracicaba, v. 41/42, n. 5, p. 44-49, 1989. 